\title{
L'Etat, l'action publique et la sociologie des champs
}

A paraître dans la Revue suisse de science politique

Vincent Dubois, Professeur à l'Université de Strasbourg, France (SAGE, UMR 7363), et Florence Gould member de l'Institute for Advanced Study, Princeton, USA

Institute for Advanced Study, School of Social Science, Einstein Drive, Princeton, NJ 08540 USA, +1 609-734-8264 v.dubois@ias.edu

Résumé : Pierre Bourdieu accorde dans son cours au collège de France une place centrale à ce qu'il appelle «les actes d'Etat». Il invite ainsi à s'intéresser aux politiques étatiques, comme il l'a fait lui-même dans son étude du logement. Cet article propose dans cette perspective d'articuler sociologie de l'Etat et sociologie de l'action publique, et explore plus précisément en ce sens quelques unes des applications possibles de la théorie des champs aux espaces de production des politiques d'Etat.

\begin{abstract}
In his course at the College de France, Pierre Bourdieu pays special attention to what he calls the 'acts of state'. By doing so he invites us to focus on state policies, as he did in his study on housing. This article follows this invitation and proposes to articulate the sociology of the state and policy sociology. More precisely, it explores in this direction some of the possible uses of Bourdieu's fields theory in the analysis of the social spaces of state policy making.
\end{abstract}

Keywords : State ; Bourdieu ; Policy ; Field analysis

Vincent Dubois a notamment publié La politique culturelle, Belin, 2012 [1999]; La vie au guichet, Economica, 2010 [1999, trad. anglaise The Bureaucrat and the poor, Ashgate, 2010] ; La politique, l'artiste et le gestionnaire, Croquant, 2012 ; La question technocratique, (dir., avec D. Dulong), PUS, 1998 ; Les mondes de l'harmonie (avec J.M. Méon et E. Pierru), La Dispute, 2009 [trad. anglaise The sociology of wind music, Ashgate, 2013]. 


\section{Introduction}

L'un des paris analytiques proposés par Pierre Bourdieu dans ses cours au collège de France (Bourdieu, 2012) consiste à saisir l'Etat au travers de ses actes. Du plus trivial acte bureaucratique (comme l'octroi d'une carte d'identité ou d'un certificat de maladie) aux « décisions » qui peuvent transformer durablement tout un secteur de la vie sociale (comme la réforme du crédit et de l'aide au logement), ces «actes d'Etat» supposent la concentration d'un ensemble de capitaux accumulés tout au long de la genèse de l'Etat pour être efficacement accomplis, et réalisent en retour la force de l'Etat. Cette force est matérielle (l'octroi de ressources) ou physique (la coercition, mise en avant notamment par Weber). Elle est aussi indissociablement symbolique, ce que l'on voie très directement dans les actes de nomination qui consistent à octroyer, certifier et officialiser un statut (celui de citoyen national ou de malade), ou, de façon plus générale, dans la diffusion sous de multiples formes d'un nomos érigé en principe légitime de représentation du monde social ${ }^{1}$. De tels actes s'inscrivent dans la structure des positions et des relations de ceux qui, en les effectuant, se fondent sur le «pouvoir de l'Etat» en même temps qu'ils lui donnent corps. Cette structure, c'est celle du champ bureaucratique, et plus largement celle des espaces où s'opère l'affrontement réglé (par l'Etat) des positions et des points de vue, et qui, comme les commissions au sein desquelles sont construits et traités les problèmes d'Etat, fondent le travail de légitimation et d'universalisation grâce auquel peut opérer la magie d'Etat.

Saisir l'Etat par ses actes : une telle perspective s'inscrit dans une tentative de dépassement de tout un ensemble de divisions scolastiques et-ou liées à la vision d'Etat, opposant la genèse à la structure, la structure au processus et à l'action, la société civile à l'Etat ou encore l'administration au politique. Dans le champ académique, et singulièrement en science politique, ces fausses oppositions sont reproduites dans une division du travail scientifique qui sépare l'Etat, l'administration et les politiques publiques en trois spécialités distinctes (Bezès, Pierru, 2012). La réflexion proposée ici prolonge cette tentative de dépassement, en envisageant les politiques publiques comme une classe particulière d' « actes d'Etat », ce qui conduit à lire de Sur l'Etat sous l'angle particulier de sa contribution à une sociologie de

\footnotetext{
${ }^{1}$ Comme lorsque la substitution de l'aide à la personne à l'aide à la pierre en matière de logement promeut l'individuel contre le collectif, et entérine la prédominance d'une logique de marché.
} 
1'action publique ${ }^{2}$. Plus précisément, nous voudrions montrer comment la théorie des champs, à l'œuvre dans les cours du collège de France comme dans toute l'œuvre de Pierre Bourdieu, peut permettre d'objectiver les systèmes de relations dans lesquels les politiques étatiques comme actes d'Etat sont élaborées et légitimées, et ainsi fonder sociologiquement une approche des politiques publiques n'oubliant pas 1'Etat qui les produit (state policies) et, pour une part, se définit par le fait de les produire (policy state) ${ }^{3}$.

\section{L'espace de production des politiques étatiques}

L'analyse des politiques publiques ne manque pas de notions pour rendre compte des systèmes de relations entre les acteurs qui contribuent à la fabrication des politiques. L'analyse des réseaux de politiques publiques (policy networks), développée d'abord aux Etats-Unis puis largement diffusée dans la littérature internationale, a donné lieu à toute une série de déclinaisons, policy community, issue network, iron triangle, advocacy coalition ou encore epistemic community ${ }^{4}$. Les travaux francophones issus de l'analyse stratégique de Michel Crozier ou de l'approche par les référentiels formulée par Pierre Muller et Bruno Jobert on quant à eux développé l'usage des notions de système d'action pour les premiers, et de secteur pour les seconds ${ }^{5}$. On ne peut se livrer ici à un examen critique de ces différentes notions. On remarquera simplement que leur foisonnement tranche singulièrement avec l'absence quasi-complète dans les travaux sur les politiques publiques du concept de champ tel que l'a construit Pierre Bourdieu. Notre proposition dans cet article est que ce concept peut pourtant s'avérer particulièrement précieux pour appréhender l'action publique d'un point de vue sociologiquement et empiriquement rigoureux.

Sa mobilisation pour reconstituer l'espace de production d'une politique repose sur un postulat qui rompt tant avec les conceptions classiques de l'action publique comme fruit d'une « volonté », d'une décision et-ou d'un cheminement rationnel qu'avec les analyses qui y voient un effet d'interaction contingent - garbage can model - (Cohen et. al., 1991) ou d'idées considérées comme matrices de l'action (Revue française de science politique, 2000).

\footnotetext{
${ }^{2}$ J'ai donné une première formulation des prémisses d'une sociologie de l'action publique telle qu'elle est conçue ici dans Dubois, 2009.

${ }^{3}$ Ce texte s'appuie sur une partie des réflexions plus amplement développées dans un travail auquel on se permet de renvoyer (Dubois, à paraître).

${ }^{4}$ Pour une présentation : voir notamment Le Galès et Thatcher, 1995.

${ }^{5}$ Crozier et Friedberg, 1977 ; Jobert et Muller, 1987.
} 
Il consiste à considérer l'action publique comme le produit des pratiques et représentations des agents qui y sont engagés, ces pratiques et représentations étant déterminées par la position objective de ces agents, et donc par la structure des relations qui les unissent. En permettant d'objectiver la structure de ces positions, des prises de positions correspondantes, et de ces relations, l'analyse en termes de champ permet de révéler ce qui fonde socialement une politique et, partant, d'en fournir une analyse sociologique.

Ce postulat conduit à formuler deux hypothèses de base. Reprenant l'un des axiomes de la sociologie des champs qui pose une relation d'homologie entre les positions et les prises de position, la première consiste à rapporter les options et orientations concurrentes dans la définition d'une politique (baisser les charges patronales ou réduire le temps de travail pour favoriser l'emploi ; préférer la route ou le chemin de fer) aux positions et intérêts de ceux qui les défendent (représentants des employeurs ou hauts fonctionnaires et experts militants du ministère de l'Emploi ; lobbyistes de l'industrie automobile ou élus écologistes). Une seconde hypothèse, plus originale et rarement mise à l'épreuve empiriquement, consiste à établir la correspondance entre le contenu d'une politique (son orientation, son style), et la structure relationnelle de l'espace des agents engagés dans sa production. C'est cette hypothèse que l'on propose de développer en considérant une politique comme l'objectivation d'un état provisoire du rapport de forces au sein du champ de luttes pour la définition légitime de cette intervention.

C'est ce dont Pierre Bourdieu a donné un exemple dans son travail sur le logement, en analysant l'espace des positions et prises de position au principe de la production des politiques en la matière (Bourdieu, 2000). Cette analyse est relativement peu citée dans les travaux mobilisant la sociologie de Bourdieu, et moins encore par les analystes des politiques publiques. Pierre Bourdieu y revient cependant très fréquemment dans Sur l'Etat, non seulement parce que les cours du collège de France coïncident avec la finalisation de cette enquête (dont les résultats ont été publiés pour la première fois en 1990 dans un numéro d'Actes de la recheche en sciences sociales), mais parce qu'au delà de son objet spécialisé qui peut paraître technique, elle est l'occasion de croiser un grand nombre des questions qui forment l'armature de sa sociologie de l'Etat. Pour en rester à l'angle qui est plus spécifiquement celui de cet article, l'étude de la dynamique des concurrences et des alliances qui aboutit à la réforme de la politique du logement nous semble constituer une référence centrale pour une sociologie positionnelle et relationnelle de l'action publique. 
Pierre Bourdieu montre comment le changement de la valeur des capitaux au sein du champ bureaucratique dans la seconde moitié des années 1970, au cours du septennat de Valéry Giscard d'Estaing, permet à une alliance conjoncturelle de jeunes techniciens sortis de l'école Polytechnique et de jeunes administrateurs des finances sortis de l'Ecole nationale d'administration de l'emporter sur les positions antérieurement établies dans le domaine des politiques du logement, fonctionnaires du ministère de l'Equipement, élus locaux et représentants des sociétés d'économie mixte. Les premiers peuvent ainsi imposer la vision « moderne » et « libérale » attachée à leur position et à leurs intérêts propres, reléguant au rang d'«archaïsmes» les conceptions des seconds. On comprend ainsi les fondements sociaux autant qu'idéologiques du déclin de «l'aide à la pierre» au profit de «l'aide à la personne », traduction technique d'une individualisation de la question du logement (le soutien financier des ménages plus que la construction de logements collectifs), révélatrice d'une première amorce du tournant néo-libéral.

Cet exemple montre que l'apport de la sociologie des champs à l'analyse de l'action publique va bien au-delà de la simple morphologie des groupes dirigeants, l'élite des « décideurs » dont il s'agirait seulement d'établir les propriétés sociales. Il s'agit bien plus de montrer ce que les propriétés des agents et la logique de leurs relations induisent en termes de prises de position, c'est-à-dire indissociablement de productions symboliques (expertises, constructions idéologiques, visions du monde légitimées) et de pratiques d'intervention (lois, règlements, décisions budgétaires, réformes, constructions institutionnelles, allocations de ressources, etc. $)^{6}$.

On voit également que cet usage va bien au-delà d'une forme un peu sophistiquée de marxisme à laquelle ses critiques réduisent souvent la sociologie des champs (voir par exemple Alexander, 1995). Ce que montre en l'occurrence la mobilisation empirique de cette sociologie, c'est que le champ de production d'une politique est rarement réductible au reflet mécanique d'un rapport de classe, et que les dominants aux positions a priori acquises n'ont pas toujours par avance partie gagnée. L'une des caractéristiques fréquentes d'un tel champ est d'être composite : des fonctionnaires dont les positions hiérarchiques, les générations, les

\footnotetext{
${ }^{6} \mathrm{La}$ thématique des «instruments de l'action publique », si elle vise à associer les dimensions symbolique ou cognitive («théorisation politique») et les usages pratiques des dispositifs ne prend pas en compte les caractéristiques sociales des groupes qui produisent et utilisent ces instruments (Lascoumes, Le Galès, 2004).
} 
corps et institutions d'appartenance sont différentes, des experts, des représentants professionnels, syndicaux, et d'intérêts divers, etc. Cela autorise des jeux d'alliances dont les retournements peuvent expliquer les changements d'orientation.

On voit donc enfin que l'objectivation de la structure d'un tel champ ne conduit pas à une vision fixiste d'un ordre immuable dont la reproduction consisterait en une reconduction à l'identique. Rendre compte de ces états successifs permet au contraire, en identifiant les déplacements des rapports de forces, de mieux comprendre des changements politiques qu'on ne saurait pas plus imputer aux «volontés » individuelles des décideurs ou à leur remplacement qu'à une simple "adaptation » des choix publics à l'évolution objective des situations sur lesquelles ils portent.

\section{Questionner l'action publique au prisme de la notion de champ}

La perspective dont on vient de résumer les principaux fondements conduit à formuler un ensemble d'interrogations qui permettent à la fois de contrôler la rigueur de l'usage du concept de champ et de le faire fonctionner comme outil pour la formulation d'hypothèses à visée empirique. On partira de cinq questions classiques de la sociologie des champs (Bourdieu 1984 ; 1992) que l'on reformulera en les appliquant à l'espace de production des politiques publiques.

Un champ se constitue en définissant un enjeu qui lui est spécifique, irréductible à ceux des autres champs. Une première question consiste donc à établir quel enjeu spécifie l'espace de production d'une politique. On peut y répondre en posant qu'il s'agit du pouvoir de régler une sphère particulière de pratiques (immigration, logement, éducation, santé, etc.), par la mobilisation de ressources (financières, juridiques, administratives, etc.) propres à une institution publique (un gouvernement national, une collectivité locale, l'Union européenne, etc.), ou liée aux pouvoirs publics (une agence mêlant public et privé, un organisme parapublic, une association financée sur fonds publics, un organisme de sécurité sociale, etc.).

Comment, en second lieu, définir et délimiter cet espace ? Comme pour n'importe quel champ, la définition de ses contours ne peut être posée a priori, mais résulte de la reconstruction réalisée dans l'enquête. Dans sa recherche sur la politique du logement, Pierre 
Bourdieu part de l'identification de ceux qu'il appelle les agents efficients, à partir des positions institutionnelles, d'une analyse réputationnelle, d'un repérage des prises de position, qui sert de base à la reconstitution systématique de l'ensemble par recoupements et compléments successifs. Dans bien des cas, les commissions ad hoc constituées autour d'un problème ou d'un domaine particulier peuvent être analysées comme l'objectivation du noyau dur du champ concerné et faire à ce titre l'objet d'une enquête particulière. C'est par exemple ce qu'on a proposé en reconstituant la formation d'un espace légitime d'élaboration des politiques culturelles en France dans les années 1960 à partir des commissions culturelles du Plan (Dubois, 2012). Ici comme ailleurs, voire ici plus qu'ailleurs, la définition des limites du champ est un enjeu de lutte puisqu'en l'occurrence « en être ou pas » correspond à l'obtention ou non d'une reconnaissance officielle à intervenir dans la régulation d'une sphère d'activité et à la potentialité d'y contribuer effectivement. On a ainsi pu établir comment et pour quelles raisons, de manière contre intuitive, les artistes ont été dans un premier temps relégués hors du champ de production des politiques culturelles.

L'existence d'un champ suppose un degré d'autonomie en deçà duquel un champ cesse de fonctionner comme tel, en étant soumis à des logiques qui lui sont extérieures. Sur l'Etat en fournit de nombreuses illustrations : loin des débats théoriques de la tradition marxiste (voir notamment Poulantzas, 1971) sur l'autonomie de l'Etat par rapport aux classes dominantes, la sociologie des champs invite à restituer empiriquement les configurations historiques des rapports de forces internes à chaque champ et des chances respectives de leurs différentes fractions à peser sur l'orientation des politiques. Elle invite de manière complémentaire à établir l'état des champs politique et bureaucratique qui détermine les possibilités d'alliance et les types d'échange avec ces différentes fractions, la régulation de leur accès différencié aux lieux de pouvoir et aux ressources publiques, la capacité ou la propension à s'imposer à elles ou à convertir leurs revendications en politique officielle. Il s'agit en d'autres termes d'établir les systèmes de relations entre différents systèmes de relations (ou champs), dans la logique d'une conception de l'Etat comme méta-champ qui, on le voit, ouvre à la recherche sociologique plus qu'à une discussion générale et abstraite sur son autonomie.

Quatrième question: quels sont les principes d'opposition qui structurent le champ de production d'une politique ? La réponse est à établir en chaque cas, mais on peut néanmoins dégager quelques principes récurrents. Le pôle des agents qui prétendent avec succès représenter l'intérêt général (e.g. grands commis de l'État, «personnalités qualifiées ») 
s'oppose à celui qui regroupe ceux qui sont rabattus vers la défense d'intérêts particuliers (e.g. représentants de syndicats professionnels, élus locaux) cette opposition pouvant recouper celle qui sépare les agents généralistes des spécialistes sectoriels. Les deux principes de légitimité concurrents de la compétence et de la légitimité politique opposent les experts aux élus, dans un jeu de délégitimation croisée des « technocrates » qui s'arrogeraient le pouvoir contre les «politiciens» avant tout soucieux de leur réélection (Dubois, Dulong, 1999). Au sein du champ bureaucratique, on observe généralement la combinaison entre des oppositions hiérarchiques verticales (Etat central vs collectivités locales, hauts fonctionnaires vs petits fonctionnaires), des oppositions fonctionnelles (e.g. administrations financières vs administrations dépensières) et des concurrences institutionnelles entre «fiefs bureaucratiques » (Allison, 1969) défendant des intérêts et des orientations divergents. Au niveau des agents individuels, cela correspond à des concurrences entre types de capitaux bureaucratiques, liées aussi à des oppositions générationnelles : expérience vs technique ; compétences internes et maîtrise des règles du jeu, juridiques ou pratiques vs compétences sectorielles, transposables hors bureaucratie.

Ces principes d'opposition se combinent à des principes de regroupement et de solidarité, comme celle, classique, qu'on observe entre membres des grands corps dans la haute administration française. Ils rendent souvent stratégiques les positions intermédiaires qui s'établissent à l'interface de ces polarités, comme les « médiateurs » évoqués plus haut dans l'analyse de Jobert et Muller, les experts multipositionnés, les syndicalistes professionnalisés proches des milieux administratifs, les mobiles d'une administration à l'autre, ou les «pantoufleurs » passés du public au privé. Ils rendent également stratégiques les espaces intermédiaires, colloques, think tanks et «lieux neutres» (Bourdieu, Boltanski, 1976) où patrons et fonctionnaires, experts et syndicalistes, ou élus de différents bords se retrouvent et élaborent un langage commun.

Quels sont, enfin - cinquième question - les produits de ces concurrences ? Il s'agit des manières politiquement légitimées de voir (objectivées par exemple dans des discours, des rapports officiels) et de traiter (matérialisées dans des dispositifs, des réformes) un «problème » ou une sphère d'activité. Ces produits sont légitimés de manière formelle, en étant endossés par un agent détenteur de l'autorité politique (un maire, un ministre) ou sanctionnés par un vote. Ils sont aussi légitimés par la logique même de fonctionnement du champ, par le respect des procédures, par la revendication d'une compétence technique ou 
scientifique, par l'accumulation d'un capital symbolique, par le recours à l'opinion publique, par la concertation plus ou moins mise en scène ou la confrontation réglée des points de vue concurrents propre à la production d'un consensus plus ou moins illusoire - à l'instar de ce qui se déroule dans les commissions dont on parlait plus haut, véritables technologies de pouvoir en tant que machines à produire de la légitimité.

\section{Conclusion}

La sociologie des champs permet ainsi de comprendre le produit (la politique publique) et, ce qui est central en la matière, les conditions et modalités de sa légitimation. On l'a vu ici en se situant, comme le fait Bourdieu dans son analyse de la politique du logement, au niveau de l'espace spécifiquement constituée autour de la production d'une politique. Cette approche n'épuise pas, loin s'en faut, les usages possibles du concept de champ qui peut également permettre, à un deuxième niveau, l'analyse des relations entre les champs ou fractions de champs mobilisés dans la conduite d'une politique. Au-delà d'un usage seulement monographique de la notion de champ, il s'agit cette fois d'établir des (systèmes de) relations entre des (systèmes de) relations. C'est plus largement en cela qu'une sociologie de l'action publique ainsi conçu rejoint la sociologie de l'Etat considéré comme un «méta champ » (Bourdieu, 2012) qui, grâce à l'accumulation des ressources disponibles dans les différents champs, peut en retour d'intervenir dans chacun d'eux.

\section{Références}

Alexander, J. C. (1995). Fin de Siècle Social Theory. London : Verso.

Allison, G. T. (1969). Conceptual Models and the Cuban Missile Crisis. American Political Science Review 63(3) : 689-718.

Bezès, Ph. et Pierru, F. (2012). État, administration et politiques publiques : les dé-liaisons dangereuses. Gouvernement et action publique 2(2) : 41-87.

Bourdieu, P. (1984). Quelques propriétés des champs. Dans Pierre Bourdieu, Questions de sociologie. Paris : Minuit (113-120).

— (1992). La logique des champs. Dans Pierre Bourdieu, Loïc Wacquant, Réponses. Paris : 
Seuil (71-90).

- (2000). L'État et la construction du marché et Le champ des pouvoirs locaux. Dans Pierre Bourdieu, Les structures sociales de l'économie. Paris : Seuil (113-180).

- (2012). Sur l'Etat. Cours au collège de France (1989-1992). Paris : Raisons d'agir-Seuil.

- et Boltanski, L. (1976). La production de l'idéologie dominante. Actes de la recherche en sciences sociale, 2-3 : 4-73.

Cohen, M.D., March, J.G. et Olsen, J.P. (1991). Le modèle du "carbage can" dans les anarchies organisées. Dans James G. March, Décision et organisation. Paris : Editions d'organisation (163-204).

Crozier, M. et Friedberg, E. (1977). L'acteur et le système. Paris : Seuil.

Dubois, V. (2009). L'action publique. Dans Antonin Cohen, Bernard Lacroix et Philippe Riutort (dir.), Nouveau manuel de science politique. Paris : La Découverte (311-325).

- (2012). La politique culturelle. Genèse d'une catégorie d'intervention publique. Paris : Belin [1 1 ère édition 1999].

- (à paraître). Les champs de l'action publique. Dans Mathieu Hilgers et Eric Mangez, dir., Utiliser la théorie des champs pour comprendre le monde social [version pré-publiée: http://halshs.archives-ouvertes.fr/halshs-00498020]

— et Dulong D. dir., (1999). La question technocratique. De l'invention d'une figure aux transformations de l'action publique. Strasbourg : Presses universitaires de Strasbourg.

Jobert, B. et Muller, P. (1987). L'Etat en action. Politiques publiques et corporatismes. Paris : PUF.

Lascoumes, P. et Le Galès, P. dir. (2004). Gouverner par les instruments. Paris : Presses de Sciences-Po.

Le Galès, P. et Thatcher, M. dir. (1995). Les réseaux de politiques publiques. Débat autour des policy networks. Paris : L'Harmattan.

Poulantzas, N. (1971). Pouvoir politique et classes sociales. Paris : Maspero.

Revue française de science politique. (2000). Les approches cognitives des politiques publiques. $50(2)$. 